\title{
Fibroblast-Activating Protein: Targeting the Roots of the Tumor Microenvironment
}

\author{
Jens T. Siveke \\ Division of Solid Tumor Translational Oncology, West German Cancer Center, University Hospital Essen, Essen, Germany; and \\ German Cancer Consortium and German Cancer Research Center, Heidelberg, Germany
}

See the associated articles on pages 1415 and 1423.

$\mathbf{T}$

he complexity of tumor biology represents an ongoing hurdle to the improvement of prognosis in many cancer entities. From a genotype-driven perspective, cancer medicine has been tremendously advanced through identification of a multitude of actionable genetic alterations. The ongoing development of sophisticated technologies and analytic methods with unprecedented resolution allows for personalized approaches using an ever-increasing armamentarium of targeted approaches. These developments have led to impressive therapeutic results for some entities and small subsets of various cancers carrying targetable alterations. However, cancer patients still too often do not benefit from such approaches, caused by the absence of actionable targets, the poorly understood lack of response despite presumably matching genotype-drug situations, the enormous inter- and intratumoral heterogeneity, and the fast development of resistance.

Notably, the tumor microenvironment (TME) has been recognized as driving the complex and heterogeneous tumor ecosystem. Indeed, there is an increasingly acknowledged connection between hard-to-treat tumors and a strong desmoplastic reaction, such as in pancreatic cancer. As one important factor, the TME plays a central role in mediating drug resistance via impaired drug delivery and activity (1). Another increasingly recognized stroma-dependent contributor to tumor progression and resistance is the remarkable phenotypic plasticity of tumors and the ability of cancer cells to undergo adaptive phenotypic changes not determined by their genome. Thus, transcriptional rewiring and change in cell state may allow cells to survive and resist harsh conditions. These transcriptional programs are highly context-dependent and include cellautonomous mechanisms but importantly also microenvironmental cues (2). Besides the role of the TME in regulating the phenotype of cancer cells, components of the TME such as the vascular and immune compartment have been identified as therapeutic targets and are now a mainstay in the therapeutic arsenal, with tremendous successes in subsets of cancer patients.

Received Aug. 6, 2018; revision accepted Aug. 6, 2018.

For correspondence or reprints contact: Jens Siveke, University Hospital Essen, Hufelandstrasse 55, Essen, 45147, Germany.

E-mail: j.siveke@dkfz-heidelberg.de

Published online Aug. 10, 2018.

COPYRIGHT (c) 2018 by the Society of Nuclear Medicine and Molecular Imaging. DOI: 10.2967/jnumed.118.214361
However, a master regulator orchestrating the structural organization of the TME, namely cancer-associated fibroblasts (CAFs), has so far proven a difficult target. CAFs are a heterogeneous and highly abundant cell population in desmoplastic tumors and have long been observed in many solid tumors yet somewhat ignored for many years. CAFs become activated via various factors including tumor-derived signals or mechanical stress and are themselves the source of extracellular matrix, cytokines, chemokines, nutrients, and other signaling factors. Although much of their precise role is still incompletely understood, recent results provide an astonishingly multifaceted array of their various functions (3). Their distinct, in part opposing, functions are highly contextual and may vary in different tumor stages and entities, thereby reflecting the ability to adapt to and influence the surrounding TME. Indeed, besides signaling factors that influence the survival of tumor cells and their acknowledged role in matrix modeling and angiogenesis, CAFs have been shown to regulate immunosurveillance by promoting or inhibiting the infiltration and accumulation of immune subsets and their spatial arrangement within tumors (3).

These complex layers of regulation will have to be considered when targeting CAFs. A marker protein of CAFs is the fibroblastactivating protein (FAP), a type II transmembrane cell surface proteinase that belongs to the dipeptidyl peptidase (DPP) family, which consists of the enzymes DPP4, FAP, DPP8, and DPP9 (4). While DPP4 and FAP are transmembrane proteases and also have extra- and intracellular soluble truncated forms, DPP8 and DPP9 are intracellular proteins. All members have a DPP activity, cleaving 2 amino acids after a proline residue off the $\mathrm{N}$ terminus of a protein. FAP, unlike the other DPP family members, has an additional endopeptidase activity that allows cleavage after a glycine-proline motif. This unique feature may be relevant in a tumor context because physiologic substrates of FAP include denatured type I collagen, $\alpha 2$-antiplasmin, and several neuropeptides. Thus, FAP can degrade proteins of the extracellular matrix, an ability that is of particular relevance in pathologic processes.

Although a role in developmental processes during embryogenesis has been described, FAP is expressed at only low levels in adult normal tissues and its function in regulating physiologic processes is not well known. Indeed, genetic depletion of FAP in normal tissues in mice led to the development of paraneoplastic syndromes, including cachexia and anemia (5), suggesting that FAP is involved in maintaining normal organ functions. During tissue remodeling during tumor development and in inflammatory conditions such as liver cirrhosis, cardiovascular diseases, and rheumatoid diseases, FAP becomes highly induced. Indeed, over $90 \%$ of epithelial cancers express FAP. Interestingly, targeting of FAP-positive CAFs in mice developing endogenous spontaneous 
tumors, such as melanoma and pancreatic cancer models, improved immunosurveillance. For example, in melanoma an improved antitumor activity of CD8-positive T cells could be observed (6), whereas in pancreatic cancer, known to be highly resistant to immune-based approaches, immune checkpoint blockade against PD-L1 and CTLA4 was more effective (7).

However, these studies of genetic depletion of FAP-positive CAFs may not entirely reflect an acute perturbation as would be the case in clinical scenarios. In a study reporting on the adoptive transfer of FAP-targeted chimeric antigen receptor T cells, depletion of CAFs led to immune-independent effects on tumor growth via remodeling of extracellular matrix, leading to a suppressed tumor angiogenesis (8). Notably, FAP can also be expressed from tumor cells, and overexpression is associated with worse prognosis in solid tumors (9). Early targeting approaches in clinical trials using talabostat, an unselective DPP inhibitor, showed insufficient clinical activity in various cancers, and thus further development was not continued. An anti-FAP antibody, sibrotuzumab, labeled with ${ }^{131} \mathrm{I}$ for therapeutic use suffered from low clearance and lack of clinical activity $(10,11)$. For years, targeting efforts against FAP remained scarce because of absence of selective and potent inhibitors with suitable properties for clinical use; however, recent studies provided promising drug development strategies for potent FAP-selective inhibitors $(12,13)$.

In 2 seminal papers in this issue of The Journal of Nuclear Medicine, Haberkorn's group now moves a big step ahead in targeting FAP for clinical use $(14,15)$. Based on a strategy to inhibit FAP enzyme activity by using 4,4-difluoroproline as described previously (16), they developed a series of quinoline-based radiopharmaceuticals for diagnostic and therapeutic use. The generated inhibitors bind human and murine FAP with rapid and almost complete internalization and, importantly, without cross-reactivity to the DPP family member DPP4, thus laying the ground for further development. By linking this FAP inhibitor (FAPI) to the chelator DOTA, they generated a theranostic tracer with favorable pharmacokinetic properties. The most promising representative of a whole tracer generation, FAPI-04, showed good pharmacokinetic properties and high affinity to FAP but not DPP4. Importantly, there was no accumulation in normal tissues, and the tracer cleared rapidly from the blood and was eliminated by the kidneys. These properties resulted in impressive images of high contrast and sensitivity with regard to tumor visualization in PET/CT scans. A high tumor uptake rate in tumor-engrafted mice and eventually in patients with metastatic epithelial cancers was described using ${ }^{68} \mathrm{Ga}-\mathrm{FAPI}-04$. Consequently, theranostic use was approached in 2 patients with metastasized breast cancer using ${ }^{90}$ Y-FAPI-04, which led to a reduction in pain symptoms at a considerably low dose.

Overall, the provided results, although still in an early developmental stage, are an impressive advancement with potentially enormous implications for targeting the microenvironment of tumors. First, the strategy to develop theranostic FAP-targeting tracers offers the possibility of testing the expression and overall suitability of a therapeutic approach in an individual patient, thus greatly facilitating personalized treatment approaches. Patients with high tumoral tracer uptake will be an obvious first-target population for a therapeutic approach. The first proof-of-concept investigation of FAPI-PET/CT in various cancers such as those of the pancreas, head and neck, colon, lung, and breast showed an equal or even improved tumor visualization with lower background in liver and brain in comparison to ${ }^{18} \mathrm{~F}$-FDG (17). This improvement may also be relevant for diagnostic purposes, as is the independence from blood sugar and the possibility of early measurements due to fast tracer kinetics.

Second, given the stroma-acting nature of this approach, the TME will potentially undergo a significant transformation with a substantial impact on tumor biology (e.g., growth, differentiation, and metabolism), as well as on the immune landscape and mechanical issues such as the drug-penetrating stromal barrier (e.g., in pancreatic cancer). Thus, extensive characterization of the TME on FAPI-induced alterations through pre- and posttreatment biopsies may ultimately be crucial to identify the most promising combination partners, be they chemotherapeutic, immune-directed, or targeted agents. Given our increasing insights into the enormous impact of CAFs on tumor progression, tissue re-(modeling), and therapeutic response, it will be exciting to explore the effect of FAP targeting using the available comprehensive analytic tools to identify the most effective combination partners and schedules.

Lastly, given the biologic role of FAP known so far, various additional clinical scenarios can be envisioned ranging from adjuvant settings (e.g., prevention of metastatic recurrence) to highly palliative and supportive indications (e.g., pain reduction). Indeed, the preclinical hypothesis that FAP is involved in formation of a premalignant or premetastatic niche (18) envisions further approaches at the preneoplastic or postoperative stage of malignant disease yet will require powerful and sophisticated preclinical models to better understand the window of opportunity.

As next steps, several questions and caveats lie ahead and will have to be addressed. FAP is a highly contextual protein with complex expression patterns and regulation. How frequently will there be a sufficiently high uptake in different tumors and within individual cancer entities, and which cancers are the most promising to approach? Further diagnostic studies with well-annotated clinical and histopathologic data and ideally concomitant tissue availability will be needed to define the most promising diagnostic and therapeutic approach. Besides the apparent role in a malignant disease context, other indications are likely of great potential. Increased FAP expression has been described in cardiovascular and rheumatoid diseases, among others, and thus studying and targeting the tissue remodeling that occurs in atherosclerosis and arthritis via FAPI will be an exciting route to follow.

Of key importance will be careful evaluation of the frequency, duration, and severity of possible toxicities. Given the preclinical findings regarding a role of FAP in normal-tissue homeostasis and the frequently encountered fragile status of patients with advanced tumors, the occurrence of early and late toxicities and paraneoplastic syndromes upon therapeutic use will need to be carefully studied. Besides safety and efficacy considerations, regulatory and reimbursement issues require increased attention for any theranostic approach to come. Besides well-established and exciting new theranostic approaches in thyroid diseases, neuroendocrine tumors, and prostate cancers, an increasing armamentarium of novel radiotheranostic agents is about to enter clinical evaluation-for example, neurotensin receptor 1 in pancreatic cancer, as recently reported (19). Thus, harmonization of requirements from the various stakeholders and regulatory agencies will be just as important as continued research along the lines of clinical development and evaluation to bring the best possible theranostic approaches to patients.

In summary, stroma targeting via the novel FAP-binding molecules reported by Haberkorn's group represents a highly promising theranostic approach with enormous potential and sets the stage to target the complex crosstalk between cancer cells, host cells, and the surrounding extracellular matrix. 


\section{DISCLOSURE}

Jens Siveke is supported by the German Research Foundation (grant SFB824/C4); the European Union's Seventh Framework Programme for research, technological development, and demonstration (FP7/CAM-PaC, grant 602783); German Cancer Aid (grant 70112505); and the German Cancer Consortium (DKTK). No other potential conflict of interest relevant to this article was reported.

\section{ACKNOWLEDGMENT}

The author apologizes to all colleagues whose work could not be included because of space constraints.

\section{REFERENCES}

1. Trédan O, Galmarini CM, Patel K, Tannock IF. Drug resistance and the solid tumor microenvironment. J Natl Cancer Inst. 2007;99:1441-1454.

2. Varga J, Greten FR. Cell plasticity in epithelial homeostasis and tumorigenesis. Nat Cell Biol. 2017;19:1133-1141.

3. LeBleu VS, Kalluri R. A peek into cancer-associated fibroblasts: origins, functions and translational impact. Dis Model Mech. 2018;11:dmm029447.

4. Hamson EJ, Keane FM, Tholen S, Schilling O, Gorrell MD. Understanding fibroblast activation protein (FAP): substrates, activities, expression and targeting for cancer therapy. Proteomics Clin Appl. 2014;8:454-463.

5. Roberts EW, Deonarine A, Jones JO, et al. Depletion of stromal cells expressing fibroblast activation protein-alpha from skeletal muscle and bone marrow results in cachexia and anemia. J Exp Med. 2013;210:1137-1151.

6. Zhang Y, Ertl HC. Depletion of FAP+ cells reduces immunosuppressive cells and improves metabolism and functions CD8+ T cells within tumors. Oncotarget. 2016;7:23282-23299.
7. Feig C, Jones JO, Kraman M, et al. Targeting CXCL12 from FAP-expressing carcinoma-associated fibroblasts synergizes with anti-PD-L1 immunotherapy in pancreatic cancer. Proc Natl Acad Sci USA. 2013;110:20212-20217.

8. Lo A, Wang LS, Scholler J, et al. Tumor-promoting desmoplasia is disrupted by depleting FAP-expressing stromal cells. Cancer Res. 2015;75:2800-2810.

9. Liu F, Qi L, Liu B, et al. Fibroblast activation protein overexpression and clinical implications in solid tumors: a meta-analysis. PLoS One. 2015;10:e0116683.

10. Scott AM, Wiseman G, Welt S, et al. A phase I dose-escalation study of sibrotuzumab in patients with advanced or metastatic fibroblast activation proteinpositive cancer. Clin Cancer Res. 2003;9:1639-1647.

11. Kloft C, Graefe EU, Tanswell P, et al. Population pharmacokinetics of sibrotuzumab, a novel therapeutic monoclonal antibody, in cancer patients. Invest New Drugs. 2004;22:39-52.

12. Poplawski SE, Lai JH, Li Y, et al. Identification of selective and potent inhibitors of fibroblast activation protein and prolyl oligopeptidase. J Med Chem. 2013;56: 3467-3477.

13. Jansen K, Heirbaut L, Cheng JD, et al. Selective inhibitors of fibroblast activation protein (FAP) with a (4-quinolinoyl)-glycyl-2-cyanopyrrolidine scaffold. ACS Med Chem Lett. 2013;4:491-496.

14. Lindner T, Loktev A, Altmann A, et al. Development of quinoline-based theranostic ligands for the targeting of fibroblast activation protein. $\mathrm{J} \mathrm{Nucl} \mathrm{Med}$. 2018;59:1415-1422.

15. Loktev A, Lindner T, Mier W, et al. A tumor-imaging method targeting cancerassociated fibroblasts. J Nucl Med. 2018;59:1423-1429.

16. Jansen K, Heirbaut L, Verkerk R, et al. Extended structure-activity relationship and pharmacokinetic investigation of (4-quinolinoyl)glycyl-2-cyanopyrrolidine inhibitors of fibroblast activation protein (FAP). J Med Chem. 2014;57:3053-3074.

17. Giesel F, Kratochwil C, Lindner T, et al. FAPI-PET/CT: biodistribution and preliminary dosimetry estimate of two DOTA-containing FAP-targeting agents in patients with various cancers. J Nucl Med. August 2, 2018 [Epub ahead of print].

18. Lo A, Li CP, Buza EL, et al. Fibroblast activation protein augments progression and metastasis of pancreatic ductal adenocarcinoma. JCI Insight. 2017;2:92232.

19. Baum RP, Singh A, Schuchardt C, et al. ${ }^{177} \mathrm{Lu}-3 \mathrm{BP}-227$ for neurotensin receptor 1-targeted therapy of metastatic pancreatic adenocarcinoma: first clinical results. J Nucl Med. 2018;59:809-814. 